\title{
Impacto de um programa de fortalecimento muscular dos membros inferiores no equilíbrio e na performance funcional de idosos institucionalizados: um estudo controlado e randomizado
}

\section{Impact of a muscle strengthening program for lower members on the balance and functional performance of institutionalized elderly: a controlled and randomized study}

\author{
(iD) Amanda Aparecida Oliveira Leopoldino ${ }^{1}$, (D) Izabella Thalita Araújo ${ }^{1}$, (D) Juliana Catarina Pires ${ }^{1}$, (D) Taís Reis de Brito ${ }^{1}$, (D) Janaine \\ Cunha Polese ${ }^{2}$, Alessandra Carvalho Bastone ${ }^{3,4}$, (D) Onésia Cristina de Oliveira Lima ${ }^{4,5}$, (D) Luana Pereira Leite Schetino ${ }^{6}$
}

\footnotetext{
${ }^{1}$ Faculdade Ciências Médicas de Minas Gerais FCMMG

2 Programa de Pós-Graduação em Ciências da Saúde, Faculdade Ciências Médicas de Minas Gerais - FCMMG

3 Escola de Educação Física, Fisioterapia Terapia Ocupacional, Universidade Federal de Minas Gerais - UFMG

${ }^{4}$ Departamento de Fisioterapia, Universidade Federal dos Vales do Jequitinhonha e Mucuri UFVJM

5 Departamento de Farmacologia, Universidade Federal de Goiás - UFG

${ }^{6}$ Faculdade de Medicina, Universidade Federal dos Vales do Jequitinhonha e Mucuri - UFVJM

\section{Correspondência}

Crystian Moraes Silva Gomes

E-mail: crystian salazar@hotmail.com
}

Submetido: 07 Agosto 2020

Aceito: 21 Outubro 2020

\section{Como citar}

Leopoldino AAO, Araújo IT, Pires JC, Brito TR, Polese JC, Bastone AC, et al. Impacto de um programa de fortalecimento muscular dos membros inferiores no equilíbrio e na performance funcional de idosos institucionalizados: um estudo controlado e randomizado. Acta Fisiatr. 2020;27(3):174-181.

DOI: $10.11606 /$ issn.2317-0190.v27i3a174188

\begin{abstract}
RESUMO
A performance funcional é maximizada através de programas de fortalecimento muscular em idosos. Contudo, poucas intervenções foram implementadas em idosos institucionalizados para avaliar os efeitos de programas de exercícios de fortalecimento muscular em desfechos como o equilíbrio, performance funcional e força muscular. Objetivo: Avaliar o impacto de um programa de fortalecimento muscular de membros inferiores no equilíbrio, performance funcional e força muscular de idosos institucionalizados. Métodos: Ensaio clínico controlado e randomizado. O equilíbrio, a performance funcional e a força muscular foram avaliados através da Escala de Equilíbrio de Berg (EEB), Marcha Tandem (MT), Short Physical Performance Battery (SPPB) e Teste do Esfigmomanômetro (TE), respectivamente. O grupo experimental (GE, $n=11$ ) participou do programa de exercícios em grupo, três vezes semanais, durante oito semanas, enquanto o grupo controle $(\mathrm{GC}, \mathrm{n}=8)$ continuou sua rotina habitual. Foram calculados os intervalos de $95 \%$ de confiança para as diferenças entre os grupos experimental e controle (experimental - controle) e entre os dois momentos de observação (pós - pré). Foi realizada análise de intenção de tratar. Resultados: $\mathrm{O}$ GE apresentou um ganho significativo em relação ao GC para a EEB (DM= 3,7 IC95\% 1,0 a 6,5), MT DM=3,5 IC95\% 0,7 a 6,2), FMEJ (DM=33,2 IC95\% 4,9 a 61,5) e FMD (DM= 37,5 IC95\% 9,6 a 65,3). Conclusão: O programa de fortalecimento muscular foi capaz de melhorar o equilíbrio, a performance funcional e a força muscular em idosos institucionalizados.
\end{abstract}

Palavras-chave: Idoso, Equilíbrio Postural, Força Muscular, Exercício Físico, Instituição de Longa Permanência para Idosos

\section{ABSTRACT}

Functional performance is maximized through muscle strengthening programs in the elderly. However, few interventions have been implemented in institutionalized elderly to assess the effects of muscle strengthening exercise programs in outcomes such as balance, functional performance and muscle strength. Objective: Assess the impact of a lower limb muscle strengthening program at the balance, functional performance and muscle strength of institutionalized elderly. Methods: Controlled, randomized clinical trial. Balance, functional performance and muscle strength were assessed using the Berg Balance Scale (BSE), Tandem Gait (MT), Short Physical Performance Battery (SPPB) and Sphygmomanometer Test (TE) respectively. The experimental group $(G E, n=11)$ participated in the group exercise program, three times a week, for eight weeks, while the control group ( $C G, n=8)$ continued their usual routine.The intervals of $95 \%$ confidence for the differences between the experimental and control groups (experimental - control) and between the two moments of observation (post pre). Intent to treat analysis was performed. Results: EG showed a significant gain in relation to CG for BSE (DM= 3.7 IC95\% 1.0 to 6.5), MT DM= 3.5 IC95\% 0.7 to 6.2), FMEJ (DM= $33.295 \% \mathrm{Cl}$ 4.9 to 61.5$)$ and $\mathrm{FMD}(\mathrm{DM}=37.595 \% \mathrm{Cl} 9.6$ to 65.3$)$. Conclusion: The muscle strengthening program was able to improve balance, functional performance and muscle strength in institutionalized elderly.

Keywords: Aged, Postural Balance, Muscle Strength, Exercise, Homes for the Aged 


\section{INTRODUÇÃO}

O envelhecimento provoca alterações fisiológicas dos sistemas vestibular, visual e somatossensorial, além de alterar funções musculoesqueléticas que acarretam em desgaste das articulações, sarcopenia e quedas, variáveis que interferem diretamente na mobilidade e no controle postural. ${ }^{1,2,3}$

Os idosos institucionalizados têm menos oportunidades de participar de atividades de vida diária (AVD) de maneira independente, e apresentam maior comprometimento da marcha e do equilíbrio em comparação aos idosos da comunidade. ${ }^{4}$ Assim, esse grupo de idosos, em especial, deve ser observado com um olhar ampliado de saúde, por meio de avaliações da capacidade funcional e da qualidade de vida. ${ }^{5}$

De acordo com um estudo anterior, a força muscular e a capacidade de desenvolver a contração muscular são fortes preditores da função cotidiana de idosos, assim para a detecção de limitações funcionais, deve se atentar para a avaliação da força muscular. ${ }^{6}$ Vale ressaltar ainda que a fraqueza muscular no envelhecimento é mais acentuada nos membros inferiores, ${ }^{7}$ e os mesmos auxiliam na realização de tarefas cotidianas importantes como levantar-se de uma cadeira, descer escadas e andar em velocidade adequada. ${ }^{8}$

Uma revisão sistemática de Liu \& Latham $^{9}$ forneceu evidências de que o treinamento de resistência progressivo é uma intervenção eficaz para melhorar a performance funcional dos idosos, incluindo a melhora do desempenho de algumas atividades simples e complexas. Ainda, o treinamento resistido foi considerado o melhor método para aumentar a força muscular e também foi eficaz na prevenção de quedas. ${ }^{10}$

Contudo, grande parte dos estudos se concentraram principalmente em idosos da comunidade, ${ }^{11}$ e a maioria deles utilizaram treinamentos multicomponentes compostos por exercícios aeróbicos, respiratórios ou de fortalecimento global, portanto não avaliaram especificamente os efeitos de um programa de fortalecimento muscular de membros inferiores. ${ }^{12}$

Considerando-se que o fisioterapeuta nas instituições de longa permanência para idosos (ILPI) tem o foco de restaurar e manter a funcionalidade dos mesmos, ${ }^{13}$ este estudo se justifica pela importância em determinar intervenções ideais para exercícios físicos necessárias para promover melhorias significativas no equilíbrio, performance funcional e força muscular de idosos institucionalizados. ${ }^{14}$

\section{OBJETIVO}

Avaliar o impacto de um programa de fortalecimento muscular de membros inferiores no equilíbrio, performance funcional e força muscular de idosos institucionalizados em ILPI.

\section{MÉTODOS}

Trata-se de um ensaio clínico e randomizado, no qual as variáveis estabelecidas foram comparadas antes e após o período de intervenção. O presente estudo foi realizado seguindo as recomendações de acordo com o protocolo checklist of information to include when reporting a randomised trial (CONSORT 2010). ${ }^{15}$
Este estudo foi aprovado pelo Comitê de Ética em Pesquisa da Faculdade Ciências Médicas de Minas Gerais e da Universidade Federal dos Vales do Jequitinhonha e Mucuri. Todos os cuidados éticos que regem pesquisas com seres humanos foram observados e respeitados, segundo a Resolução 466/2012 regulamentada pelo Conselho Nacional de Saúde.

\section{Amostra do estudo}

Foram recrutados idosos residentes de uma ILPI da cidade de Belo Horizonte-MG e idosos residentes de duas ILPI da cidade de Diamantina-MG. Os idosos selecionados foram divididos aleatoriamente, por meio de sorteio com envelope selado, em dois grupos, 50\% para compor o grupo controle (GC) e $50 \%$ para o grupo experimental (GE).

Para o cálculo amostral a análise de poder revelou que um tamanho de amostra de oito participantes seria necessário em cada grupo. Considerou-se um nível de significância de 0,05, teste de duas caudas, poder de $80 \%$, um efeito de 1,56 , baseado no efeito do exercício no SPPB no estudo de Bastone \& Filho ${ }^{6}$ e perda de $20 \%$.

Os critérios de inclusão consistiram em: idosos institucionalizados em ILPI públicas, com idade igual ou superior a 60 anos, capazes de realizar o teste "Timed Up and Go" (TUG), ${ }^{17}$ de forma independente com ou sem uso de dispositivo de auxílio para a marcha. Foram excluídos os idosos que apresentaram déficit cognitivo e que mediante avaliação médica apresentaram doença cardíaca, respiratória ou metabólica descompensada, ou alguma condição de saúde que poderia comprometer de forma significativa o desempenho do idoso nos testes da avaliação.

Previamente à avaliação, os idosos incluídos no estudo assinaram o Termo de Consentimento Livre e Esclarecido (TCLE) e posteriormente responderam a um questionário de dados sociodemográficos e clínicos para caracterização da amostra. As avaliações, antes e após o período de intervenção, foram realizadas por duas pesquisadoras "cegadas" em relação à alocação dos participantes.

Todos os testes utilizados na avaliação apresentam boa confiabilidade, validade bem estabelecida e já foram traduzidos para a população brasileira. Os testes estão descritos a seguir.

\section{Métodos de Avaliação}

Este estudo foi aprovado pelo Comitê de Ética em Pesquisa da Faculdade Ciências Médicas de Minas Gerais e da Universidade Federal dos Vales do Jequitinhonha e Mucuri. Todos os cuidados éticos que regem pesquisas com seres humanos foram observados e respeitados, segundo a Resolução 466/2012 regulamentada pelo Conselho Nacional de Saúde.

Foram recrutados idosos residentes de uma ILPI da cidade de Belo Horizonte-MG e idosos residentes de duas ILPI da cidade de Diamantina-MG. Os idosos selecionados foram divididos aleatoriamente, por meio de sorteio com envelope selado, em dois grupos, $50 \%$ para compor o grupo controle (GC) e $50 \%$ para o grupo experimental (GE). 
Para o cálculo amostral a análise de poder revelou que um tamanho de amostra de oito participantes seria necessário em cada grupo. Considerou-se um nível de significância de 0,05, teste de duas caudas, poder de $80 \%$, um efeito de 1,56 , baseado no efeito do exercício no SPPB no estudo de Bastone \& Filho ${ }^{6}$ e perda de $20 \%$.

Os critérios de inclusão consistiram em: idosos institucionalizados em ILPI públicas, com idade igual ou superior a 60 anos, capazes de realizar o teste "Timed Up and Go" (TUG), ${ }^{17}$ de forma independente com ou sem uso de dispositivo de auxílio para a marcha. Foram excluídos os idosos que apresentaram déficit cognitivo e que mediante avaliação médica apresentaram doença cardíaca, respiratória ou metabólica descompensada, ou alguma condição de saúde que poderia comprometer de forma significativa o desempenho do idoso nos testes da avaliação.

Previamente à avaliação, os idosos incluídos no estudo assinaram o Termo de Consentimento Livre e Esclarecido (TCLE) e posteriormente responderam a um questionário de dados sociodemográficos e clínicos para caracterização da amostra. As avaliações, antes e após o período de intervenção, foram realizadas por duas pesquisadoras "cegadas" em relação à alocação dos participantes.

Todos os testes utilizados na avaliação apresentam boa confiabilidade, validade bem estabelecida e já foram traduzidos para a população brasileira. Os testes estão descritos a seguir.

\section{Escala de Equilíbrio de Berg (EEB)}

Trata-se de um teste que avalia o desempenho do equilíbrio funcional com base em 14 itens comuns à vida diária. ${ }^{18}$ Alguns dos itens avaliam o indivíduo parado; girando 360 graus; em apoio unipodal; levantando-se de uma cadeira, entre outros. A pontuação máxima é de 56 pontos e cada item possui uma escala com cinco alternativas que variam de 0 (incapaz de realizar) a 4 pontos (realiza a tarefa de forma independente). ${ }^{19}$

O teste é simples, de fácil aplicação e seguro para avaliar pacientes idosos, requerendo apenas um relógio e uma régua como equipamentos. ${ }^{18} \mathrm{~A}$ análise da pontuação é feita da seguinte forma: $0-36$ pontos: $100 \%$ risco para quedas; $37-44$ pontos: locomoção segura, com recomendação de auxílio para marcha; 45-56 pontos: locomoção segura. ${ }^{19}$

\section{Marcha Tandem (MT)}

Esse teste avalia o equilíbrio dinâmico. Consiste em solicitar que o indivíduo deambule em uma linha reta, de forma que o calcâneo de um pé fique a frente dos artelhos do outro pé. Considera-se bom desempenho quando o indivíduo consegue dar mais de dez passos sobre a linha reta e desempenho fraco quando são dados menos de dois passos sob a linha reta. ${ }^{20}$

\section{Short Physical Performance Battery (SPPB)}

O SPPB avalia a performance funcional, através de três componentes: velocidade de caminhada, equilíbrio em pé e o desempenho do sentar e levantar da cadeira, em que cada componente é pontuado de 0 a 4 (pontuação máxima). 0 equilíbrio foi avaliado com o indivíduo em pé nas posições lado a lado, suporte semi-tandem e suporte tandem por 10 segundos.

Na caminhada cronometrada o indivíduo foi orientado a caminhar no ritmo normal. O menor tempo das duas tentativas de andar por um percurso de 4 metros foi considerado. $\mathrm{Na}$ atividade de sentar e levantar da cadeira, a mesma foi posicionada de acordo com a necessidade do participante levantar-se dela com os braços cruzados no peito, e repetir 5 vezes o mais rápido possível. Indivíduos incapazes de completar qualquer tarefa foram pontuados 0 .

A soma dos três componentes é pontuada com um intervalo possível de 0 a 12 , sendo que 12 indica o mais alto grau de funcionalidade. ${ }^{21,22}$

\section{Teste do Esfigmomanômetro (TE)}

A força muscular especificamente dos extensores de joelho e dorsiflexores foi avaliada bilateralmente, tendo em vista que são os maiores produtores de potência na marcha em indivíduos idosos. ${ }^{23}$

$\mathrm{O}$ teste foi realizado com o esfigmomanômetro insuflado a $100 \mathrm{mmHg}$, mantendo a válvula fechada, para que possíveis dobras fossem retiradas. Logo após, a pressão foi reduzida até $20 \mathrm{mmHg}$ e a válvula fechada novamente para evitar vazamentos. $^{24}$ O posicionamento do teste foi realizado baseado no estudo de Benfica et al. ${ }^{25}$

\section{Grupo Experimental (GE)}

O GE participou de um programa de fortalecimento muscular para membros inferiores, em grupos de quatro idosos, por um período de oito semanas, com frequência de três vezes semanais e duração média de uma hora por sessão. O programa foi conduzido por outras três pesquisadoras que não tinham acesso aos dados da avaliação, e o mesmo seguiu as recomendações de treinamento resistido segundo as diretrizes do American College of Sports Medicine e da American Heart Association. ${ }^{26}$ Os exercícios propostos estão descritos no Quadro 1.

\section{Grupo Controle (GC)}

Os idosos incluídos no GC participaram das mesmas avaliações no início e no final do estudo, semelhante ao GE. Porém, o GC recebeu apenas orientações, não participou de nenhum exercício e foi instruído a continuar suas atividades de rotina diária habitual.

\section{Análise estatística}

A análise dos dados foi realizada por uma pesquisadora que desconhecia a distribuição dos grupos. Foram comparadas as diferenças entre os valores obtidos na avaliação inicial e final e entre grupos.

As variáveis numéricas foram apresentadas como média \pm desvio-padrão e as variáveis categóricas, como frequências absolutas e relativas. Foram calculados os intervalos de confiança 95\% (IC95\%) para as diferenças entre os GE e GC (experimental - controle) e entre os dois momentos de observação (pós - pré). 
Quadro 1. Programa de Exercícios

I. Sentar e levantar de uma cadeira

II. Ficar na ponta dos pés e dos calcanhares
Paciente foi solicitado a sentar-se e levantar-se de uma cadeira com encosto, sem a ajuda das mãos, 20 vezes no início e 20 vezes no final da sessão.

Paciente de pé, com as duas mãos apoiadas em uma cadeira, foi solicitado a ficar na ponta dos pés (flexão plantar) e em seguida sobre os calcanhares (dorsiflexão) sustentando o peso corporal em ambas as situações. Foram realizadas três séries de dez repetições para cada movimento.

III. Exercício para fortalecimento de quadríceps

Paciente sentado em cadeira com encosto, foi solicitado a estender o joelho, com uso de tornozeleira. Foram realizadas três séries de oito repetições em cada membro.

IV. Exercício para fortalecimento dos isquiotibiais

Paciente de pé, deve realizar flexão de joelho, com uso de tornozeleira. Foram realizadas três séries de oito repetições em cada membro.

V. Elevação da perna

Paciente de pé, mantendo o joelho em extensão e se apoiando com a mão em uma barra de apoio, deve realizar flexão, extensão, adução e abdução de quadril, com uso de tornozeleira. Foram realizadas três séries de oito repetições com cada um dos membros inferiores.

Nota: Em relação aos exercícios III, IV e V, o peso utilizado será estabelecido individualmente. Inicialmente, a resistência foi determinada como $80 \%$ de uma repetição máxima. ${ }^{27}$ Quando os exercícios foram executados corretamente e sem sinais de cansaço ao final da terceira série, a resistência era aumentada.

As variáveis numéricas foram apresentadas como média \pm desvio-padrão e mediana (10 quartil - 3ㅇquartil) e as variáveis categóricas, como frequências absolutas e relativas. A comparação de médias pré e pós intervenção foi avaliada pelo teste de Wilcoxon e a comparação entre os grupos, pelo teste de Mann-Whitney. Foi considerado nível de significância de 5\% e a análise foi realizada no software $\mathrm{R}$ versão 4.0.0.

Em relação à análise por intenção de tratar, para os pacientes do grupo controle foi repetido o valor obtido na linha de base. Foi realizada como se os sujeitos tivessem recebido o tratamento (ou a condição de controle) que lhes foram atribuídas inicialmente.

\section{RESULTADOS}

Dos 22 indivíduos selecionados inicialmente, três participantes do GC não foram reavaliados devido à problemas de saúde e razão pessoal (Figura 1). O número total de sessões realizadas no período de treinamento foi de 24 e os participantes tiveram uma frequência média de 20 sessões (83,3\%).

A idade média da amostra foi de $(77,5 \pm 8,7)$ no $G E$ e $(70,4$ $\pm 6,3)$ no GC. Em relação às demais características dos 19 idosos que finalizaram o estudo, houve diferença entre o GE e o GC somente no tempo de institucionalização que foi significativamente maior no GC (Tabela 1 ).

Verificou-se que o GE obteve uma melhora significativa nos testes $\operatorname{EEB}(52,1 \pm 3,3)$, MT $(5,9 \pm 4,3)$, SPPB $(9,0 \pm 1,8)$, FMEJ $(142,3 \pm 41,2)$ e $\operatorname{FMD}(96,5 \pm 37,7)$, enquanto o $G C$ não apresentou diferença significativa entre os valores obtidos nos testes após o período de oito semanas (Tabela 2).

Em comparação com os valores da linha de base, os escores do EEB, MT, SPPB, FMEJ e FMD foram significativamente melhores após 8 semanas de exercício no GE. Já no grupo controle não houve mudanças estatisticamente significativas nos valores dos testes EEB, SPPB, FMEJ da linha de base até o final do estudo, enquanto os valores da $\operatorname{MT}(-0,3 \pm 1,3)$ e da FMD $(-16,4 \pm 20,4)$ apresentaram declínio com o tempo (Tabela 2).

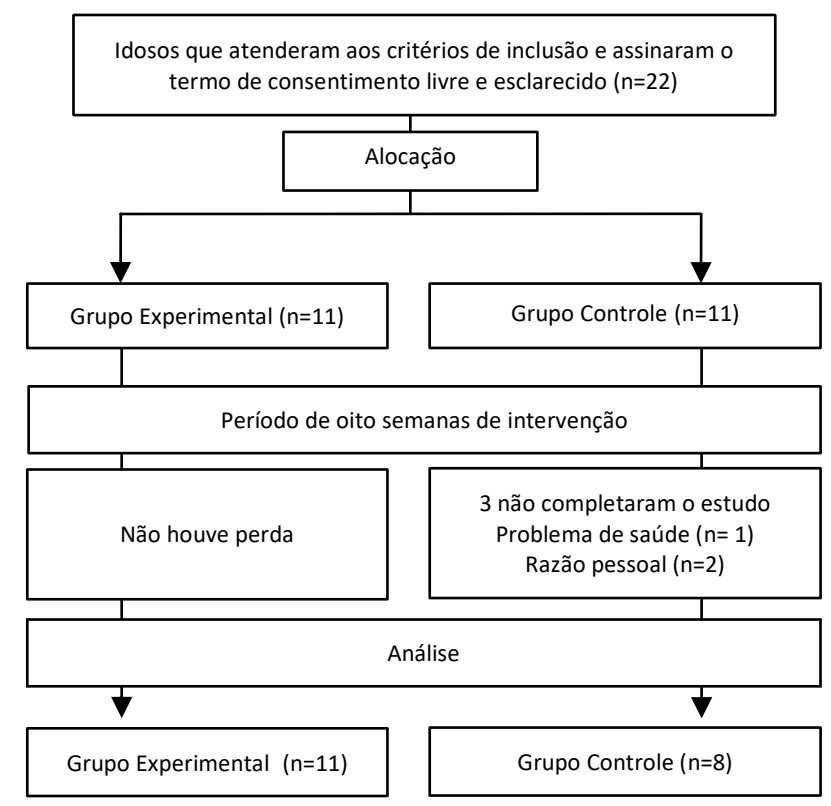

Figura 1. Fluxograma dos participantes do ensaio clínico randomizado

Tabela 1. Características sociodemográficas e de funcionalidade dos participantes $(n=19)$

\begin{tabular}{lcc}
\hline & GE $(\mathbf{n}=\mathbf{1 1})$ & $\mathbf{G C}(\mathbf{n}=\mathbf{8})$ \\
\hline Feminino & $8(72,7)$ & $7(87,5)$ \\
Masculino & $3(27,3)$ & $1(12,5)$ \\
Idade & $77,5 \pm 8,7$ & $70,4 \pm 6,3$ \\
Tempo de institucionalização & $5,9 \pm 4,0$ & $12,9 \pm 9,7$ \\
EEB & $47,9 \pm 4,0$ & $46,5 \pm 5,5$ \\
MT & $2,7 \pm 3,5$ & $1,5 \pm 2,3$ \\
SPPB & $7,0 \pm 1,9$ & $5,9 \pm 2,2$ \\
FMEJ (mmHg) & $125,5 \pm 23,3$ & $100,5 \pm 34,5$ \\
FMD (mmHg) & $55,9 \pm 21,9$ & $49,1 \pm 12,4$ \\
\hline
\end{tabular}

GE: grupo experimental; GC: grupo controle; SPPB: Short Physical Performance Battery; EEB: Escala de Equilibrio de Berg; MT: Marcha Tandem; FMEJ: Força Muscular de Extensores de Joelho; FMD: Força Muscular de Dorsiflexores; mmHg: milímetros de mercurio 
Observa-se que o GE apresentou um ganho significativo em relação ao GC para a EEB ( $\mathrm{DM}=3,7 \mathrm{IC} 95 \% 1,0$ a 6,5$)$, MT (DM=3,5 IC95\% 0,7 a 6,2), FMEJ (DM=33,2 IC95\% 4,9 a 61,5) e FMD (DM=37,5 IC95\% 9,6 a 65,3).
Pode-se afirmar que os idosos alocados no GE apresentaram melhora do equilíbrio e performance funcional, além do aumento significativo na força muscular de extensores de joelho e dorsiflexores.

Tabela 2. Média (DP) dos grupos, diferença média (DP) intra grupos e diferença média (IC 95\%, p) entre grupos

\begin{tabular}{|c|c|c|c|c|c|c|c|}
\hline \multirow{3}{*}{ Desfecho } & \multicolumn{4}{|c|}{ GRUPOS } & \multirow{2}{*}{\multicolumn{2}{|c|}{$\begin{array}{c}\text { Diferença intra grupos } \\
\begin{array}{c}\text { Semana } 8 \text { menos } \\
\text { Baseline }\end{array}\end{array}$}} & \multirow{3}{*}{$\begin{array}{c}\text { Diferença inter grupos } \\
\begin{array}{c}\text { Semana } 8 \text { menos } \\
\text { Baseline }\end{array} \\
\text { Exp menos Con }\end{array}$} \\
\hline & \multicolumn{2}{|c|}{ Baseline } & \multicolumn{2}{|c|}{ Semana 8} & & & \\
\hline & $\begin{array}{c}\text { GE } \\
(n=11)\end{array}$ & $\begin{array}{c}G C \\
(n=8)\end{array}$ & $\begin{array}{c}\text { GE } \\
(n=11)\end{array}$ & $\begin{array}{c}\text { GC } \\
(n=8)\end{array}$ & GE & GC & \\
\hline EEB & $47,9 \pm 4,0$ & $46,5 \pm 5,5$ & $52,1 \pm 3,3$ & $47,0 \pm 4,9$ & $\begin{array}{c}4,2 \pm 4,0 \\
{[1,5 ; 6,9]}\end{array}$ & $\begin{array}{c}0,5 \pm 1,2 \\
{[-0,4 ; 1,3]}\end{array}$ & $\begin{array}{c}3,7 \\
{[1,0 ; 6,5]}\end{array}$ \\
\hline MT & $2,7 \pm 3,5$ & $1,5 \pm 2,3$ & $5,9 \pm 4,3$ & $1,3 \pm 2,1$ & $\begin{array}{c}3,2 \pm 3,9 \\
{[0,5 ; 5,8]}\end{array}$ & $\begin{array}{c}-0,3 \pm 1,3 \\
{[-1,1 ; 0,6]}\end{array}$ & $\begin{array}{c}3,5 \\
{[0,7 ; 6,2]}\end{array}$ \\
\hline SPPB & $7,0 \pm 1,9$ & $5,9 \pm 2,2$ & $9,0 \pm 1,8$ & $6,5 \pm 1,9$ & $\begin{array}{c}2,0 \pm 1,82,0 \\
{[0,8 ; 3,2]}\end{array}$ & $\begin{array}{c}0,6 \pm 1,0 \\
{[-0,1 ; 1,3]}\end{array}$ & $\begin{array}{c}1,4 \\
{[0,0 ; 2,7]}\end{array}$ \\
\hline FMEJ & $125,5 \pm 23,3$ & $100,5 \pm 34,5$ & $142,3 \pm 41,2$ & $84,1 \pm 46,8$ & $\begin{array}{l}16,8 \pm 39,0 \\
{[-9,4 ; 43,0]}\end{array}$ & $\begin{array}{l}-16,4 \pm 20,4 \\
{[-30,1 ;-2,7]}\end{array}$ & $\begin{array}{c}33,2 \\
{[4,9 ; 61,5]}\end{array}$ \\
\hline FMD & $55,9 \pm 21,9$ & $49,1 \pm 12,4$ & $96,5 \pm 37,7$ & $52,3 \pm 15,1$ & $\begin{array}{c}40,6 \pm 41,3 \\
{[12,9 ; 68,4]}\end{array}$ & $\begin{array}{c}3,2 \pm 5,1 \\
{[-0,3 ; 6,6]}\end{array}$ & $\begin{array}{c}37,5 \\
{[9,6 ; 65,3]}\end{array}$ \\
\hline
\end{tabular}

GE: grupo experimental; GC: grupo controle; SPPB: Short Physical Performance Battery; EEB: Escala de Equilibrio de Berg; MT: Marcha Tandem; FMEJ: Força Muscular de Extensores de Joelho; FMD: Força Muscular de Dorsiflexores; $\mathrm{mmHg}$ : milimetros de mercurio

\section{DISCUSSÃO}

Os resultados deste estudo demonstraram que o programa de fortalecimento muscular promoveu melhora significativa em relação ao equilíbrio, performance funcional e força muscular, avaliados através dos testes EEB, MT, SPPB e TE respectivamente. Observou-se através da diferença intragrupos que GE apresentou melhor desempenho em todos os testes realizados comparado ao GC após o período de oito semanas.

Em relação à caracterização da amostra, os dados indicam uma disparidade entre os sexos, em que há predominância do sexo feminino em ambos os grupos GE e GC. Tal fato corresponde aos achados de Lacerda et al. $^{28}$ em que o sexo feminino correspondeu à cerca de $67 \%$ do público residente em ILPI no estado de Minas Gerais. Já o tempo de institucionalização foi significativamente maior no GC $(12,9 \pm$ $9,7)$, comparado ao $\mathrm{GE}(5,9 \pm 4,0)$. Isso pode justificar o fato do GC apresentar um pior desempenho em todos os valores dos testes na da linha de base.

Lini et. al $^{29}$ citam em seu estudo que com o avançar da idade, aumentam as situações de dependência que a ILPI impõe aos idosos, dessa forma subentende-se que quanto mais prolongado o tempo de institucionalização maior será o grau de isolamento e maiores serão as consequências físicas e sociais acarretadas aos idosos. ${ }^{30} \mathrm{O}$ equilíbrio funcional avaliado através da EEB apresentou diferença significativa na pontuação dos idosos do GE comparado ao GC, com uma diferença intergrupos de (DM=3,7 IC95\% 1,0 a 6,5) após o período de oito semanas.

Porém, segundo um estudo de Conradsson et. al ${ }^{31}$ uma mudança de 8 pontos na EEB seria necessária para revelar uma mudança significativa na função entre os idosos que são institucionalizados. Portanto, é necessário um maior período de intervenção, com exercícios específicos com o propósito de melhorar o equilíbrio e consequentemente a pontuação na EEB.

Além disso, segundo Viveiro et. $\mathrm{al}^{32}$ pontuações menores que 47 na EEB em idosos institucionalizados indicam um maior risco de quedas. Apesar do GE não apresentar uma mudança significativa após a intervenção, nota-se que ambos os grupos GE e GC apresentaram pontuações acima de 47, portanto um baixo risco de quedas.

Com relação à $\mathrm{MT}$, também utilizada para avaliar o equilíbrio, a diferença inter-grupos foi de (DM= 3,5 IC95\% 0,7 a $6,2)$ após o período de oito semanas, refletindo um melhor desempenho no grupo de idosos que realizaram a intervenção.

A população idosa geralmente apresenta um desempenho fraco neste teste, ${ }^{20}$ o que corrobora com os nossos achados, e poucos estudos utilizaram a MT como método de avaliação em ensaios clínicos. Kim et al. ${ }^{33}$ relataram que não há um protocolo padronizado baseado em diretrizes para validar o teste de MT embora o mesmo continue sendo amplamente utilizado.

Do ponto de vista clínico, os escores baixos na escala SPPB têm um valor preditivo no desempenho das AVD e na perda de mobilidade. ${ }^{3}$ Os idosos tanto do GE como do GC apresentaram inicialmente uma pontuação baixa no SPPB nos valores da linha de base, considerando que pontuações iguais ou menores que 10 indicam deficiência de mobilidade. ${ }^{34}$

Após o período de oito semanas, a diferença inter grupos foi de $1,4[0,0 ; 2,7]$, e segundo Perera et. al ${ }^{35}$ uma mudança substancial de 1,0 pontos no SPPB é clinicamente relevante. Complementar a isso, uma revisão sistemática ${ }^{26}$ observou que após a participação de idosos em programas de exercícios físicos obteve-se melhora da marcha e da performance funcional medida com a SPPB, porém o estudo foi realizado com idosos frágeis residentes na comunidade.

Desta forma, é importante que mais pesquisas incluam programas de exercícios em idosos institucionalizados, com 
foco de melhorar a performance funcional dos mesmos e comprovar tais achados através do SPPB.

$\mathrm{O}$ presente estudo demonstrou um aumento significativo na força muscular dos dorsiflexores e extensores de joelho nos idosos do GE. Esse dado é corroborado por Ribeiro et al. ${ }^{36}$ que observaram através de um treinamento de força de 6 semanas, utilizando bandas elásticas, a melhora da força muscular de flexores plantares e dorsiflexores, além de melhora do equilíbrio e da mobilidade funcional em idosos institucionalizados.

O mesmo estudo aponta que o declínio da força com o envelhecimento é mais acentuado no tornozelo do que no joelho, principalmente nos músculos dorsiflexores. A força de dorsiflexão é citada como um preditor significativo e independente no desempenho do sentado para de pé, fortemente associada ao equilíbrio e capacidade funcional. ${ }^{36}$

No entanto, são escassos na literatura os estudos que avaliam o impacto de programas constituídos exclusivamente por exercícios de fortalecimento muscular no equilíbrio de idosos institucionalizados.

Um estudo de Sahin et al. ${ }^{37}$ examinou os efeitos de exercícios resistidos de diferentes intensidades realizados 3 vezes na semana, durante oito semanas em idosos frágeis institucionalizados. Entretanto, este estudo implementou exercícios resistidos tanto de membros superiores como de membros inferiores, além das variáveis de treinamento e métodos de avaliação serem diferentes comparados ao presente estudo.

Em um estudo prospectivo randomizado de Strasser et al. ${ }^{38}$ observaram que o treinamento resistido com faixas elásticas a longo prazo, melhorou a força muscular em pessoas muito idosas $(82,4 \pm 6,0$ anos). Enquanto que a suplementação nutricional adicional não foi capaz de melhorar ainda mais os efeitos obtidos pelo treinamento isolado.

Outro estudo similar, Hofmann et al. ${ }^{39}$ avaliaram também o treinamento resistido com faixa elástica e suplementação nutricional, e verificou-se que o treinamento de força melhora o desempenho físico e qualidade muscular mesmo em mulheres institucionalizadas muito idosas. Porém, ambos os estudos apresentam diferenças comparados ao presente estudo, pois utilizaram outros métodos de avaliação como testes isocinéticos, absorciometria de raio- $X$, teste de suporte de cadeira e dinamômetros, além de protocolos de exercício diferentes e a suplementação nutricional como complementar.

O declínio cognitivo é um dos principais motivos para institucionalização de idosos e, com frequência, contribui para o aumento da dependência na ILPI. ${ }^{40}$ Além dos benefícios na performance funcional, o estudo de Rugbeer et al. ${ }^{41}$ comprovou que exercícios físicos em grupo, numa frequência de 3 vezes por semana, resultou em melhora na saúde mental de idosos residentes em instituições de longa permanência.

Mais um estudo de Bossers et al. ${ }^{42}$ constatou que uma combinação de treinamento aeróbio e de força é mais eficaz do que o treinamento apenas aeróbio para retardar o declínio cognitivo e motor em idosos institucionalizados com demência.

Em suma, pode-se dizer que o programa de exercícios utilizado neste estudo é de alta aplicabilidade clínica, por ser de baixo custo e fácil acesso e pode ser implementado em qualquer ILPI, pois necessita de acessórios simples, tais como caneleiras e cadeiras.

Diante dos efeitos positivos do programa de fortalecimento muscular no equilíbrio, na performance funcional e na força muscular dos indivíduos do GE deste estudo, intervenções semelhantes devem ser implementadas por fisioterapeutas nas ILPI, para promover melhora da funcionalidade e da qualidade de vida desses idosos.

Futuros estudos sobre os efeitos do fortalecimento muscular em idosos institucionalizados devem ser conduzidos utilizando-se um tamanho maior de amostra, maiores períodos de treinamento e acompanhamento, além de outros métodos de avaliação.

Este estudo apresentou algumas limitações, pois em relação à caracterização da amostra o GC apresentou um tempo de institucionalização significativamente maior que o $\mathrm{GE}$, o que poderia afetar os resultados desse grupo. Além disso, a duração do treinamento foi relativamente curta e nenhum acompanhamento a longo prazo foi realizado, o que pode limitar a generalização dos resultados.

\section{CONCLUSÃO}

O programa de fortalecimento muscular de membros inferiores foi capaz de melhorar o equilíbrio, a performance funcional, além de aumentar a força muscular de extensores de joelhos e dorsiflexores em grupos de idosos institucionalizados.

\section{AGRADECIMENTOS}

Agradecemos o apoio financeiro do Programa Institucional de Bolsas de Iniciação Científica (PROBIC).

\section{REFERENCIAS}

1. Araujo LB, Moreira NB, Villegas ILP, Loureiro APC, Israel $\mathrm{VL}$, Gato SA, et al. Investigação dos saberes quanto à capacidade funcional e qualidade de vida em idosas institucionalizadas, sob a ótica da CIF. Acta Fisiatr. 2015; 3(22):111-7. Doi: https://doi.org/10.5935/01047795.20150022

2. Pereira NMR, Araya MJPM, Scheicher ME. Analysis of correlation between instruments for evaluation of postural balance in institutionalized elderly. MOJ Gerontol Ger. 2019; 4 (2): 69-72. Doi: https://doi.org/10.15406/mojgg.2019.04.00181

3. Rogan S, Radlinger L, Baur H, Schmidtbleicher D, Bie RA, Bruin ED. Sensory-motor training targeting motor dysfunction and muscle weakness in long-term care elderly combined with motivational strategies: a single blind randomized controlled study. Eur Rev Aging Phys Act. 2016;13(4). Doi: https://doi.org/10.1186/s11556016-0164-0

4. Gustavson AM, Falvey JR, Jankowski CM, Stevens-Lapsley JE. Public Health Impact Of Frailty: Role Of Physical Therapists. J Frailty Aging. 2017;6(1):2-5. Doi: https://doi.org/10.14283/ ifa.2017.1 
5. Ferreira LMBM, Roig JJ, Andrade FLJP, Oliveira NPD, Araújo JRT, Lima KC. Prevalência de quedas e avaliação da mobilidade em idosos institucionalizados. Rev Bras. Geriatr Gerontol. 2016;19(6):995-1003. Doi: https://doi.org/10.1590/1981-22562016019.160034

6. Van Roie E, Verschueren SM, Boonen S, Kennis E, Coudyzer W, Delecluse C. Force-velocity characteristics of the knee extensors: an indication of the risk for physical frailty in elderly women. Arch Phys Med Rehabil. 2011;92(11):1827-32.

Doi:

https://doi.org/10.1016/i.apmr.2011.05.022

7. Ticinesi A, Meschi T, Narici MV, Lauretani F, Maggio M. Muscle ultrasound and sarcopenia in older individuals: a clinical perspective. J Am Med Dir Assoc. 2017;18(4):290300. Doi: https://doi.org/10.1016/j.jamda.2016.11.013

8. Soares AV, Marcelino E, Maia KC, Borges JNG. Relation between functional mobility and dynapenia in institutionalized frail elderly. Einstein (São Paulo). 2017; 15(3):278-82. Doi: https://doi.org/10.1590/s167945082017ao3932

9. Liu CJ, Latham NK. Progressive resistance strength training for improving physical function in older adults. Cochrane Database Syst Rev. 2009;2009(3):CD002759. Doi: https://doi.org/10.1002/14651858.CD002759.pub2

10. Sousa N, Mendes R. Comparison of effects of resistance and multicomponent training on falls prevention in institutionalized elderly women. J Am Geriatr Soc. 2015;63(2):396-7. Doi: https://doi.org/10.1111/jgs.13280

11. Arrieta H, Rezola-Pardo C, Zarrazquin I, Echeverria I, Yanguas JJ, Iturburu $\mathrm{M}$, et al. A multicomponent exercise program improves physical function in long-term nursing home residents: A randomized controlled trial. Exp Gerontol. 2018;103:94-100. Doi: https://doi.org/10.1016/i.exger.2018.01.008

12. Arrieta H, Rezola-Pardo C, Kortajarena M, Hervás G, Gil J, Yanguas JJ, et al. The impact of physical exercise on cognitive and affective functions and serum levels of brain-derived neurotrophic factor in nursing home residents: a randomized controlled trial. Maturitas. 2020;131:72-77.

Doi:

https://doi.org/10.1016/j.maturitas.2019.10.014

13. Brett $L$, Noblet $T$, Jorgensen $M$, Georgiou $A$. The use of physiotherapy in nursing homes internationally: $A$ systematic review. PLoS One. 2019;14(7):e0219488. Doi: https://doi.org/10.1371/journal.pone.0219488

14. Brett L, Traynor V, Stapley P. Effects of physical exercise on health and well-being of individuals living with a dementia in nursing homes: a systematic review. J Am Med Dir Assoc. 2016 Feb;17(2):104-16. Doi: https://doi.org/10.1016/i.jamda.2015.08.016

15. Moher D, Hopewell S, Schulz KF, Montori V, Gøtzsche PC, Devereaux PJ, et al. CONSORT 2010 explanation and elaboration: updated guidelines for reporting parallel group randomised trials. BMJ. 2010;340:c869. Doi: https://doi.org/10.1136/bmj.c869

16. Bastone Ade C, Jacob Filho W. Effect of an exercise program on functional performance of institutionalized elderly. J Rehabil Res Dev. 2004;41(5):659-68. Doi: https://doi.org/10.1682/jrrd.2003.01.0014
17. Bohannon RW. Reference values for the timed up and go test: a descriptive meta-analysis. J Geriatr Phys Ther. 2006; 29(2):64-8. Doi: http://dx.doi.org/10.1519/00139143-200608000-00004

18. Miyamoto ST, Lombardi Junior I, Berg KO, Ramos LR, Natour J. Brazilian version of the Berg balance scale. Braz J Med Biol Res. 2004;37(9):1411-21. Doi: http://dx.doi.org/10.1590/s0100-879x2004000900017

19. Gomes CS, Rangel GMB, Sant'Ana MEGS, Santos MAT, Fraga WLA, Soares EV, et al. Efeitos do treinamento sensório motor por meio de dispositivos ecoeficientes sobre a capacidade funcional e equilíbrio em idosos: ensaio clínico controlado. Persp Online: Biol Saúde. 2018;8(28):42-58.

Doi: https://doi.org/10.25242/886882820181652

20. $\mathrm{Hu} \mathrm{M}$, Woollacott M. Balance evaluation, training and rehabilitation of frail fallers. Rev Clin Gerontol. 1996;6(1):85-99.

Doi: https://doi.org/10.1017/S0959259800004524

21. Nakano MM. Versão Brasileira da Short Physical Performance Battery-SPPB: adaptação cultural e estudo da confiabilidade [Dissertação]. Campinas; Universidade Estadual de Campinas - UNICAMP; 2007.

22. Guralnik JM, Ferrucci L, Simonsick EM, Salive ME, Wallace RB. Lower-extremity function in persons over the age of 70 years as a predictor of subsequent disability. N Engl J Med. 1995; 332(9):556-61. Doi: https://doi.org/10.1056/NEJM199503023320902

23. Silva SM, Corrêa FI, Silva PFC, Silva DFT, Lucareli PRG, Corrêa JCF. Validation and reliability of a modified sphygmomanometer for the assessment of handgrip strength in Parkinson's disease. Braz J Phys Ther. 2015;19(2):137-45. Doi: https://doi.org/10.1590/bjptrbf.2014.0081

24. Helewa A, Goldsmith $\mathrm{CH}$, Smythe $\mathrm{HA}$. The modified sphygmomanometer - an instrument to measure muscle strength: a validation study. J Chron Dis. 1981; 34(7):353361. Doi: https://doi.org/10.1016/0021-9681(81)90073-4

25. Benfica PD, Aguiar LT, Brito SA, Bernadino LH, TeixeiraSalmela LF, Faria CD. Reference values for muscle strength: a systematic review with a descriptive metaanalysis. Braz J Phys Ther. 2018;22(5):355-69. Doi: https://doi.org/10.1016/i.bjpt.2018.02.006

26. Giné-Garriga $M$, Roqué-Fíguls $M$, Coll-Planas $L$, SitjàRabert $M$, Salvà $A$. Physical exercise interventions for improving performance-based measures of physical function in community-dwelling, frail older adults: a systematic review and meta-analysis. Arch Phys Med Rehabil. 2014;95(4):753-769.e3. Doi: http://dx.doi.org/10.1016/i.apmr.2013.11.007

27. Avers $D$, Brown M. White paper: Strength training for the older adult. J Geriatr Phys Ther. 2009;32(4):148-52.

28. Lacerda TTB, Horta NC, Souza MCMR, Oliveira TRPR, Marcelino KGS, Ferreira QN. Caracterização das Instituições de longa permanência para idosos da região metropolitana de Belo Horizonte. Rev Bras Geriatr Gerontol. 2017;20(6):743-54. Doi: https://doi.org/10.1590/1981-22562017020.170014 
29. Lini EV, Portella MR, Doring M. Fatores associados à institucionalização de idosos: um estudo caso-controle. Rev Bras Geriatr Gerontol. 2016; 19(6):1004-14. Doi: https://doi.org/10.1590/1981-22562016019.160043

30. Martins GB, Medeiros FD. Avaliação da capacidade funcional de idosos institucionalizados e não institucionalizados [texto na Internet]. Tubarão (SC): Unisul; c2005 [citado 2020 Ago 30]. Disponível em: http://fisio-

tb.unisul.br/Tccs/06b/gilmara/artigogilmara.pdf

31. Conradsson $M$, Lundin-Olsson L, Lindelöf $N$, Littbrand $H$, Malmqvist L, Gustafson $Y$ et al. Berg balance scale: intrarater test-retest reliability among older people dependent in activities of daily living and living in residential care facilities. Phys Ther. 2007;87(9):1155-63. Doi: https://doi.org/10.2522/ptj.20060343

32. Viveiro LAP, Gomes GCV, Bacha JMR, Carvas Junior N, Kallas ME, Reis M, et al. Reliability, Validity, and Ability to Identity Fall Status of the Berg Balance Scale, Balance Evaluation Systems Test (BESTest), Mini-BESTest, and Brief-BESTest in Older Adults Who Live in Nursing Homes. J Geriatr Phys Ther. 2019;42(4):E45-E54. Doi: https://doi.org/10.1519/JPT.0000000000000215

33. Kim KJ, Gimmon Y, Millar J, Schubert MC. Using inertial sensors to quantify postural sway and gait performance during the Tandem Walking Test. Sensors (Basel). 2019;19(4):751. Doi: https://doi.org/10.3390/s19040751

34. Vasunilashorn S, Coppin AK, Patel KV, Lauretani F, Ferrucci $L$, Bandinelli $S$, et al. Use of the short physical performance battery score to predict loss of ability to walk 400 meters: analysis from the InCHIANTI study. J Gerontol A Biol Sci Med Sci. 2009;64A(2):223-9. Doi: https://doi.org/10.1093/gerona/gln022

35. Perera S, Mody SH, Woodman RC, Studenski SA. Meaningful change and responsiveness in common physical performance measures in older adults. J Am Geriatr Soc. 2006;54(5):743-9. Doi: https://doi.org/10.1111/j.1532-5415.2006.00701.x

36. Ribeiro F, Teixeira F, Brochado G, Oliveira J. Impact of low cost strength training of dorsi- and plantar flexors on balance and functional mobility in institutionalized elderly people. Geriatr Gerontol Int. 2009;9(1):75-80. Doi: https://doi.org/10.1111/j.1447-0594.2008.00500.x
37. Sahin UK, Kirdi N, Bozoglu E, Meric A, Buyukturan G, Ozturk $A$, et al. Effect of low-intensity versus highintensity resistance training on the functioning of the institutionalized frail elderly. Int J Rehabil Res. 2018;41(3):211-7.

Doi: https://doi.org/10.1097/MRR.0000000000000285

38. Strasser EM, Hofmann M, Franzke B, Schober-Halper B, Oesen $S$, Jandrasits $W$, et al. Strength training increases skeletal muscle quality but not muscle mass in old institutionalized adults: a randomized, multi-arm parallel and controlled intervention study. Eur J Phys Rehabil Med. 2018;54(6):921-33. Doi: https://doi.org/10.23736/S19739087.18.04930-4

39. Hofmann M, Schober-Halper B, Oesen S, Franzke B, Tschan $\mathrm{H}$, Bachl N, et al. Effects of elastic band resistance training and nutritional supplementation on muscle quality and circulating muscle growth and degradation factors of institutionalized elderly women: the Vienna Active Ageing Study (VAAS). Eur J Appl Physiol. 2016;116 (5):885-97. Doi: https://doi.org/10.1007/s00421-016$\underline{3344-8}$

40. Menezes AV, Aguiar AS, Alves EF, Quadros LB, Bezerra PP. Efetividade de uma intervenção fisioterapêutica cognitivo-motora em idosos institucionalizados com comprometimento cognitivo leve e demência leve. Ciênc Saúde Coletiva. 2016;21(11):3459-67. Doi: https://dx.doi.org/10.1590/1413812320152111.17892015

41. Rugbeer N, Ramklass S, Mckune A, van Heerden J. The effect of group exercise frequency on health related quality of life in institutionalized elderly. Pan Afr Med J. 2017;26:35.

Doi: https://dx.doi.org/10.11604/pamj.2017.26.35.10518

42. Bossers WJ, van der Woude LH, Boersma F, Hortobágyi T, Scherder EJ, van Heuvelen MJ. A 9-week aerobic and strength training program improves cognitive and motor function in patients with dementia: a randomized, controlled trial. Am J Geriatr Psychiatry. 2015;23(11):1106-16. Doi: 